\title{
Polyharmonic functions with negative coefficients
}

\author{
K. Al-Shaqsia,*, R. Al-Khal ${ }^{b}$ \\ a Department of Information Technology, Nizwa College of Technology, Ministry of Manpower, Sultanate of Oman. \\ ${ }^{b}$ Department of Mathematics, Sciences College, University of Dammam, Dammam, Saudi Arabia.
}

\begin{abstract}
A $2 p$ times continuously differentiable complex-valued mapping $F=u+i v$ in a domain $\mathcal{D} \subset \mathbb{C}$ is polyharmonic if $F$ satisfies the polyharmonic equation $\underbrace{\Delta \cdots \Delta}_{\mathrm{p}} \mathrm{F}=0$, where $\mathrm{p} \in \mathbb{N}^{+}$and $\Delta$ represents the complex Laplacian operator. The main aim of this paper is to introduce a subclasses of polyharmonic mappings. Coefficient conditions, distortion bounds, extreme points, of the subclasses are obtained. (C)2017 All rights reserved.
\end{abstract}

Keywords: Univalent functions, polyharmonic mappings, extreme points.

2010 MSC: 30C45, 30C50.

\section{Introduction}

Let $\mathcal{A}$ denote the class of functions of the form

$$
f(z)=z+\sum_{n=2}^{\infty} a_{n} z^{n},
$$

which are analytic in the open unite disk $\mathbb{U}=\{z: z \in \mathbb{C}$ and $|z|<1\}$. Also let $\mathcal{S}$ denote the subclasses of $\mathcal{A}$ consisting of functions which are univalent in $\mathbb{U}$. A continuous mapping $f=\mathfrak{u}+\mathfrak{i v}$ is a complex-valued harmonic mapping in a domain $\mathcal{D} \subset \mathbb{C}$ if both $u$ and $v$ are real harmonic in $\mathcal{D}$, i.e., $\Delta u=\Delta v=0$, where $\Delta$ is the complex Laplacian operator

$$
\Delta=4 \frac{\partial^{2}}{\partial z \partial \bar{z}}:=\frac{\partial^{2}}{\partial x^{2}}+\frac{\partial^{2}}{\partial y^{2}}
$$

In any simply connected domain $\mathcal{D} \subset \mathbb{C}$ we can write $f=h+\bar{g}$, where $h$ and $g$ are analytic in $\mathcal{D}$. We call $h$ the analytic part and $g$ the co-analytic part of $f$. A necessary and sufficient condition for $f$ to be locally univalent and sense-preserving in $\mathcal{D}$ is that $\left|h^{\prime}(z)\right|>\left|g^{\prime}(z)\right|$ for all $z \in \mathcal{D}$. See Clunie and Sheil-Small [2].

\footnotetext{
*Corresponding author

Email addresses: khalifa.alshaqsi@nct.edu.om (K. Al-Shaqsi), ralkhal@uod.edu.sa (R. Al-Khal)
} 
Denote by $\mathcal{H}$ the class of functions $f=h+\bar{g}$ that are harmonic univalent and sense-preserving in the unit disk $\mathbb{U}=\{z:|z|<1\}$ for which $f(0)=h(0)=f_{z}(0)-1=0$. For $f=h+\bar{g} \in \mathcal{S}_{\mathcal{H}}$ we may express the analytic functions $h$ and $g$ as

$$
h(z)=z+\sum_{n=2}^{\infty} a_{n} z^{n}, \quad g(z)=\sum_{n=1}^{\infty} b_{n} z^{n}, \quad\left|b_{1}\right|<1 .
$$

Observe that $\mathcal{H}$ reduces to $\mathcal{S}$, the class of normalized univalent analytic functions, if the co-analytic part of $f$ is zero. Denote by $\mathcal{H} \mathcal{S}^{*}$ and $\mathcal{H} \mathcal{C}$ the subclasses of $\mathcal{H} \mathcal{S}$ consisting of functions $f$ that map $\mathbb{U}$ onto starlike and convex domain, respectively.

In 1984 Clunie and Sheil-Small [2] investigated the class $\mathcal{H}$ as well as its geometric subclasses and obtained some coefficient bounds. Since then, there has been several related papers on $\mathcal{H}$ and its subclasses such that Silverman [6], Silverman and Silvia [7], and Jahangiri [3] studied the harmonic univalent functions.

\section{Preliminaries}

A continuous complex-valued mapping $F$ in $\mathcal{D}$ is biharmonic if the Laplacian of $F$ is harmonic, i.e., $F$ satisfies the equation $\Delta(\Delta \mathrm{F})=0$. It can be shown that in a simply connected domain $\mathcal{D}$, every biharmonic mapping has the representation

$$
\mathrm{F}(z)=\mathrm{G}_{1}(z)+|z|^{2} \mathrm{G}_{2}(z),
$$

where both $\mathrm{G}_{1}$ and $\mathrm{G}_{2}$ are harmonic in $\mathcal{D}$.

More generally, a complex-valued mapping $\mathrm{F}$ of a domain $\mathcal{D}$ is called polyharmonic (or $p$-harmonic) if $\mathrm{F}$ satisfies the equation $\Delta^{\mathrm{p} F}=\Delta\left(\Delta^{\mathrm{p}-1} \mathrm{~F}\right)=0$ for $\mathrm{p} \in \mathbb{N}^{+}$.

In a simply connected domain, a mapping $F$ is polyharmonic if and only if $F$ has the following representation:

$$
\mathrm{F}(z)=\mathrm{H}(z)+\overline{\mathrm{G}(z)}=\sum_{k=1}^{\mathrm{p}}|z|^{2(k-1)} \mathrm{J}_{\mathrm{p}-\mathrm{k}+1}(z),
$$

where $\Delta \mathrm{J}_{\mathrm{p}-\mathrm{k}+1}(z)=0$ and for each $\mathrm{J}_{\mathrm{p}-\mathrm{k}+1}=\mathrm{h}_{\mathrm{p}-\mathrm{k}+1}+\overline{\mathrm{g}}_{\mathrm{p}-\mathrm{k}+1},(\mathrm{k} \in\{1, \ldots, \mathrm{p}\})$ is harmonic in $\mathcal{D}$, where

$$
h_{p-k+1}(z)=\sum_{n=1}^{\infty} a_{n, p-k+1} z^{n}, \quad g_{p-k+1}(z)=\sum_{n=1}^{\infty} b_{n, p-k+1} z^{n}, \quad\left(a_{1, p}=1,\left|b_{1, p}\right|<1\right) .
$$

Denote by $\mathcal{H}_{\mathrm{p}}^{0}\left(\mathrm{~b}_{1, \mathrm{p}}=0, \mathrm{a}_{1, \mathrm{p}-\mathrm{k}+1}=\mathrm{b}_{1, \mathrm{p}-\mathrm{k}+1}=0\right)$ the subclass of $\mathcal{H}_{\mathrm{p}}$ the class of function $\mathrm{F}$ of the form (2.1) that are harmonic, univalent, and sense-preserving in the unit disk. Obviously, if $p=1$ and $\mathrm{p}=2, \mathrm{~F}$ is harmonic and biharmonic, respectively. Biharmonic mappings arise in a lot of physical situations, particularly in fluid dynamics and elasticity problems, and have many important applications in engineering and biology.

In [5], Qiao and Wang introduced the class $\mathcal{H} \mathcal{S}_{p}$ of polyharmonic mappings F given by (2.1) satisfying the condition

$$
\sum_{k=1}^{p} \sum_{n=2}^{\infty}[2(k-1)+n]\left(\left|a_{n, p-k+1}\right|+\left|b_{n, p-k+1}\right|\right) \leqslant 1-\left|b_{1,1}\right|-\sum_{k=2}^{p}(2 k-1)\left(\left|a_{1, p-k+1}\right|+\left|b_{1, p-k+1}\right|\right),
$$

where $0 \leqslant\left|b_{1,1}\right|+\sum_{k=2}^{p}(2 k-1)\left(\left|a_{1, p-k+1}\right|+\left|b_{1, p-k+1}\right|\right)<1$, and the subclass $\mathcal{H} \mathcal{C}_{p}$ of $\mathcal{H} \mathcal{S}_{p}$, where

$$
\sum_{k=1}^{p} \sum_{n=2}^{\infty}\left[2(k-1)+n^{2}\right]\left(\left|a_{n, p-k+1}\right|+\left|b_{n, p-k+1}\right|\right) \leqslant 1-\left|b_{1,1}\right|-\sum_{k=2}^{p}(2 k-1)\left(\left|a_{1, p-k+1}\right|+\left|b_{1, p-k+1}\right|\right) .
$$


The classes of all mappings $F$ in $\mathcal{H} \mathcal{S}_{\mathrm{p}}^{0}$ which are of the form (2.1), and subject the conditions (2.3) and (2.4) are denoted by $\mathcal{H} \mathcal{S}_{\mathrm{p}}^{0}, \mathcal{H} \mathcal{C}_{\mathrm{p}}^{0}$, respectively. lows:

Now we introduce new classes of polyharmonic mappings, denoted by $\mathcal{H} \mathcal{S}_{p}(\alpha)$ and $\mathcal{H C}_{p}(\alpha)$ as fol-

Denote by $\mathcal{H} \mathcal{S}_{\mathfrak{p}}(\alpha)$ the class of all functions of the form (2.1) that satisfy the condition

$$
\frac{\partial}{\partial \theta}\left(\arg \mathrm{F}\left(r e^{i \theta}\right)\right) \geqslant \alpha, \quad(0 \leqslant \alpha<1,|z|=r<1) .
$$

Also, denote by $\mathcal{H} \mathcal{T}_{p}(\alpha)$ the subclass of $\mathcal{H} \mathcal{S}_{p}(\alpha)$ such that the functions $H$ and $G$ in $F=H+\bar{G}$ are of the form:

$$
\begin{aligned}
& \mathrm{H}(z)=z-\sum_{n=2}^{\infty}\left|\mathrm{a}_{n, 1}\right| z^{n}-\sum_{k=2}^{p} \sum_{n=2}^{\infty}|z|^{2(k-1)}\left|\mathrm{a}_{n, p-k+1}\right| z^{n}, \\
& \mathrm{G}(z)=\sum_{n=2}^{\infty}\left|\mathrm{b}_{n, 1}\right| \overline{z^{n}}+\sum_{k=2}^{p} \sum_{n=1}^{\infty}|z|^{2(k-1)}\left|\mathrm{b}_{n, p-k+1}\right| \overline{z^{n}} .
\end{aligned}
$$

Also denote by $\mathcal{H}_{\mathfrak{p}}(\alpha)$ the class of all functions of the form (2.1) that satisfy the condition

$$
\frac{\partial}{\partial \theta}\left[\arg \left(\frac{\partial}{\partial \theta} \mathrm{F}\left(r e^{i \theta}\right)\right)\right] \geqslant \alpha, \quad(0 \leqslant \alpha<1,|z|=r<1) .
$$

Note that:

(i) if $\alpha=0$, then the classes $\mathcal{H} \mathcal{S}_{p}(\alpha)$ and $\mathcal{H C}_{p}(\alpha)$ reduce to the classes $\mathcal{H} \mathcal{S}_{p}$ and $\mathcal{H C}_{p}$ introduced and studied by Qiao and Wang [5];

(ii) if $p=1$, then the classes $\mathcal{H S}_{\mathfrak{p}}(\alpha)$ and $\mathcal{H C}_{p}(\alpha)$ reduce to the classes $\mathcal{H} \mathcal{S}(\alpha)$ and $\mathcal{H} \mathcal{C}(\alpha)$ studied by Jahangiri [4];

(iii) if $\alpha=0, p=1$, then the classes $\mathcal{H S}_{p}(\alpha)$ and $\mathcal{H C}_{p}(\alpha)$ reduce to the classes $\mathcal{H} \mathcal{S}$ and $\mathcal{H} \mathcal{C}$ studied by Avci and Zlotiewicz [1].

\section{Main results}

Theorem 3.1. Let $\mathrm{F}$ be given by (2.1) and

$$
\begin{aligned}
& \sum_{k=1}^{p} \sum_{n=2}^{\infty}\left\{\left[\frac{2(k-1)+n-\alpha}{1-\alpha}\right]\left|a_{n, p-k+1}\right|+\left[\frac{2(k-1)+n+\alpha}{1-\alpha}\right]\left|b_{n, p-k+1}\right|\right\} \\
& \leqslant 1-\frac{1+\alpha}{1-\alpha}\left|b_{1,1}\right|-\sum_{k=2}^{p}\left\{\frac{2 k-1-\alpha}{1-\alpha}\left|a_{1, p-k+1}\right|+\frac{2 k-1+\alpha}{1-\alpha}\left|b_{1, p-k+1}\right|\right\},
\end{aligned}
$$

where $0 \leqslant \frac{1+\alpha}{1-\alpha}\left|\mathrm{b}_{1,1}\right|+\sum_{\mathrm{k}=2}^{\mathrm{p}}\left\{\frac{2 \mathrm{k}-1-\alpha}{1-\alpha}\left|\mathrm{a}_{1, \mathrm{p}-\mathrm{k}+1}\right|+\frac{2 \mathrm{k}-1+\alpha}{1-\alpha}\left|\mathrm{b}_{1, \mathrm{p}-\mathrm{k}+1}\right|\right\}<1$. Then $\mathrm{F}$ is univalent and sense preserving in $\mathbb{U}$ and $\mathrm{F} \in \mathcal{H} \mathcal{S}_{\mathrm{p}}(\alpha)$.

Proof. First, we note that $F$ is locally univalent and sense-preserving in $\mathbb{U}$. This is because

$$
\begin{aligned}
\left|H^{\prime}(z)\right| & >1-\sum_{k=2}^{p}\left((2 k-1)\left|a_{1, p-k+1}\right| r^{2(k-1)}-\sum_{k=1}^{p} \sum_{n=2}^{\infty}[2(k-1)+n]\left|a_{n, p-k+1}\right| r^{2(k-1)+n-1}\right. \\
& >1-\sum_{k=2}^{p}(2 k-1)\left|a_{1, p-k+1}\right|-\sum_{k=1}^{p} \sum_{n=2}^{\infty}[2(k-1)+n]\left|a_{n, p-k+1}\right|
\end{aligned}
$$




$$
\begin{aligned}
& \geqslant 1-\sum_{k=2}^{p} \frac{2 k-1-\alpha}{1-\alpha}\left|a_{1, p-k+1}\right|-\sum_{k=1}^{p} \sum_{n=2}^{\infty} \frac{2(k-1)+n+1-\alpha}{1-\alpha}\left|a_{n, p-k+1}\right| \\
& \geqslant \sum_{k=1}^{p} \sum_{n=1}^{\infty}\left[\frac{2(k-1)+n+\alpha}{1-\alpha}\right]\left|b_{n, p-k+1}\right| \\
& \geqslant \sum_{k=1}^{p} \sum_{n=1}^{\infty}[2(k-1)+n]\left|b_{n, p-k+1}\right| \\
& >\sum_{k=1}^{p} \sum_{n=1}^{\infty} 2(k-1)+n\left|b_{n, p-k+1}\right| r^{2(k-1)+n-1} \geqslant\left|G^{\prime}(z)\right| .
\end{aligned}
$$

To show that $F$ is univalent in $\mathbb{U}$ we notice that for $\left|z_{1}\right| \leqslant\left|z_{2}\right|<1$, and by (3.1), we have

$$
\begin{aligned}
\left|\mathrm{F}\left(z_{1}\right)-\mathrm{F}\left(z_{2}\right)\right| \geqslant & \left|\mathrm{H}\left(z_{1}\right)-\mathrm{H}\left(z_{2}\right)\right|-\left|\overline{\mathrm{G}\left(z_{1}\right)-\mathrm{G}\left(z_{2}\right)}\right| \\
= & \left|\left(z_{1}-z_{2}\right)+\sum_{k=1}^{p} \sum_{n=2}^{\infty} a_{n, p-k+1}\left(z_{1}^{n}-z_{2}^{n}\right)\right|-\left|\sum_{k=1}^{p} \sum_{n=1}^{\infty} \overline{b_{n, p-k+1}}\left(\overline{z_{1}^{n}}-\overline{z_{2}^{n}}\right)\right| \\
\geqslant & \left|z_{1}-z_{2}\right|\left\{1-\left|\sum_{n=2}^{\infty} a_{n, p} \frac{z_{1}^{n}-z_{2}^{n}}{z_{1}-z_{2}}+\sum_{n=1}^{\infty} \overline{b_{n, p}} \frac{\overline{z_{1}^{n}}-\overline{z_{2}^{n}}}{z_{1}-z_{2}}\right|\right. \\
& \left.-\left|\sum_{k=2}^{p}\left(\sum_{n=1}^{\infty} a_{n, p-k+1} \frac{\left|z_{1}\right|^{2(k-1)}\left(z_{1}^{n}-z_{2}^{n}\right)}{z_{1}-z_{2}}+\sum_{n=1}^{\infty} \frac{b_{n, p-k+1}}{\left.z_{1}\right|^{2(k-1)}\left(\overline{z_{1}^{n}}-\overline{z_{2}^{n}}\right)}\right)\right|\right\} \\
\geqslant & \left|z_{1}-z_{2}\right|\left(1-\left|b_{1,1}\right|-\left|z_{2}\right| \sum_{n=2}^{\infty} n\left(\left|a_{n, p}\right|+\left|b_{n, p}\right|\right)\right. \\
& \left.-\left|z_{2}\right| \sum_{k=2}^{p} \sum_{n=1}^{\infty}(2(k-1)+n)\left(\left|a_{n, p-k+1}\right|+\left|b_{n, p-k+1}\right|\right)\right) \\
\geqslant & \left|z_{1}-z_{2}\right|\left(1-\left|b_{1,1}\right|-\left|z_{2}\right| \sum_{n=2}^{\infty}\left\{\frac{n-\alpha}{1-\alpha}\left|a_{n, p}\right|+\frac{n+\alpha}{1-\alpha}\left|b_{n, p}\right|\right\}\right. \\
& \left.-\left|z_{2}\right| \sum_{k=2}^{p} \sum_{n=1}^{\infty}\left\{\left(\frac{2(k-1)+n-\alpha}{1-\alpha}\right)\left|a_{n, p-k+1}\right|+\left(\frac{2(k-1)+n+\alpha}{1-\alpha}\right)\left|b_{n, p-k+1}\right|\right\}\right) \\
& \geqslant\left|z_{1}-z_{2}\right|\left(1-\left|b_{1,1}\right|\right)\left(1-\left|z_{2}\right|\right)>0 .
\end{aligned}
$$

Consequently, $\mathrm{F}$ is univalent in $\mathbb{U}$.

Now we show that $F \in \mathcal{H} \mathcal{S}_{p}(\alpha)$. According to the condition (2.4) we only need to show that if (3.1) holds, then

$$
\frac{\partial}{\partial \theta}\left(\arg \mathrm{F}\left(r e^{i \theta}\right)\right)=\mathfrak{I}\left(\frac{\partial}{\partial \theta} \log \mathrm{F}\left(r e^{i \theta}\right)\right)=\mathfrak{R}\left(\frac{z \mathrm{H}^{\prime}(z)-\overline{z \mathrm{G}^{\prime}(z)}}{\mathrm{H}(z)+\mathrm{G}(z)}\right) \geqslant \alpha,
$$

where $z=r e^{i \theta}, 0 \leqslant \theta<2 \pi, 0 \leqslant r<1$, and $0 \leqslant \alpha<1$.

Using the fact that $\Re \mathcal{R} \geqslant \alpha$ if and only if $|1-\alpha+w| \geqslant|1+\alpha-w|$, it suffices to show that

$$
|A(z)+(1-\alpha) B(z)|-|A(z)-(1+\alpha) B(z)| \geqslant 0,
$$

where $\mathrm{B}(z)=\mathrm{H}(z)+\overline{\mathrm{G}(z)}$ and $z \mathrm{H}^{\prime}(z)-\overline{z \mathrm{G}^{\prime}(z)}$. 
Substituting for $\mathrm{B}(z)$ and $A(z)$ in (3.2),

$$
\begin{aligned}
& |A(z)+(1-\alpha) B(z)|-|A(z)-(1+\alpha) B(z)| \\
& =\left|(1-\alpha) \mathrm{H}(z)+z \mathrm{H}^{\prime}(z)+\overline{(1-\alpha) \mathrm{G}(z)-z \mathrm{G}^{\prime}(z)}\right| \\
& -\left|(1+\alpha) \mathrm{H}(z)-z \mathrm{H}^{\prime}(z)+\overline{(1+\alpha) \mathrm{G}(z)+z \mathrm{G}^{\prime}(z)}\right| \\
& \geqslant \sum_{k=1}^{p} \sum_{n=1}^{\infty}[2(k-1)+n+1-\alpha]\left|a_{n, p-k+1}\right||z|^{2(k-1)+n} \\
& -\sum_{k=1}^{p} \sum_{n=1}^{\infty}[2(k-1)+n-1+\alpha]\left|b_{n, p-k+1}\right||z|^{2(k-1)+n} \\
& +\sum_{k=1}^{p} \sum_{n=1}^{\infty}[2(k-1)+n-1-\alpha]\left|a_{n, p-k+1}\right||z|^{2(k-1)+n} \\
& -\sum_{k=1}^{p} \sum_{n=1}^{\infty}[2(k-1)+n+1+\alpha]\left|b_{n, p-k+1}\right||z|^{2(k-1)+n} \\
& \geqslant(2-\alpha)|z|+\sum_{k=2}^{p}[2(k-1)+2-\alpha]\left|a_{1, p-k+1}\right|+\sum_{k=1}^{p} \sum_{n=2}^{\infty}[2(k-1)+n+1-\alpha]\left|a_{n, p-k+1}\right||z|^{2(k-1)+n} \\
& -\alpha\left|b_{1,1}\right||z|-\sum_{k=2}^{p}[2(k-1)+\alpha]\left|b_{1, p-k+1}\right||z|^{2(k-1)+1} \\
& -\sum_{k=1}^{p} \sum_{n=2}^{\infty}[2(k-1)+n-1+\alpha]\left|b_{n, p-k+1}\right||z|^{2(k-1)+n} \\
& -\alpha|z|+\sum_{k=2}^{p}[2(k-1)-\alpha]\left|a_{1, p-k+1}\right|+\sum_{k=1}^{p} \sum_{n=2}^{\infty}[2(k-1)+n-1-\alpha]\left|a_{n, p-k+1}\right||z|^{2(k-1)+n} \\
& -(2+\alpha)\left|b_{1,1}\right||z|-\sum_{k=2}^{p}[2(k-1)+2+\alpha]\left|b_{1, p-k+1}\right||z|^{2(k-1)+1} \\
& -\sum_{k=1}^{p} \sum_{n=2}^{\infty}[2(k-1)+n+1+\alpha]\left|b_{n, p-k+1}\right||z|^{2(k-1)+n} \\
& \geqslant 2(1-\alpha)|z|\left\{1-\frac{1+\alpha}{1-\alpha}\left|b_{1,1}\right|-\sum_{k=2}^{p} \frac{(2 k-1-\alpha)}{1-\alpha}\left|a_{1, p-k+1}\right||z|^{2 k+n-3}\right. \\
& -\sum_{k=2}^{p} \frac{(2 k-1+\alpha)}{1-\alpha}\left|b_{1, p-k+1}\right||z|^{2 k+n-3}-\sum_{k=1}^{p} \sum_{n=2}^{\infty} \frac{[2(k-1)+n-\alpha]}{1-\alpha}\left|a_{n, p-k+1}\right||z|^{2 k+n-3} \\
& \left.-\sum_{k=1}^{p} \sum_{n=2}^{\infty} \frac{[2(k-1)+n+\alpha]}{1-\alpha}\left|b_{n, p-k+1}\right||z|^{2 k+n-3}\right\} \\
& \geqslant 2(1-\alpha)|z|\left\{1-\frac{1+\alpha}{1-\alpha}\left|b_{1,1}\right|-\sum_{k=2}^{p} \frac{(2 k-1-\alpha)}{1-\alpha}\left|a_{1, p-k+1}\right|-\sum_{k=2}^{p} \frac{(2 k-1+\alpha)}{1-\alpha}\left|b_{1, p-k+1}\right|\right. \\
& \left.-\sum_{k=1}^{p} \sum_{n=2}^{\infty} \frac{[2(k-1)+n-\alpha]}{1-\alpha}\left|a_{n, p-k+1}\right|-\sum_{k=1}^{p} \sum_{n=2}^{\infty} \frac{[2(k-1)+n+\alpha]}{1-\alpha}\left|b_{n, p-k+1}\right|\right\} \geqslant 0 \text {, by (3.1). }
\end{aligned}
$$


The starlike polyharmonic mappings

$$
F(z)=\sum_{k=1}^{p}|z|^{2(k-1)}\left\{\sum_{n=1}^{\infty}\left\{\frac{1-\alpha}{[2(k-1)+n-\alpha]} x_{n, p-k+1} z^{n}+\frac{1-\alpha}{[2(k-1)+n+\alpha]} \overline{y_{n, p-k+1}} \overline{z^{n}}\right\}\right\},
$$

where $\sum_{k=1}^{p}\left\{\sum_{n=1}^{\infty}\left\{\left|x_{n, p-k+1}\right|+\left|y_{n, p-k+1}\right|\right\}\right\}=1$, show that the coefficient bound given by (3.1) is sharp. The functions of form (3.3) are in $\mathcal{H} \mathcal{S}_{\mathrm{p}}(\alpha)$ because

$$
\begin{aligned}
& \sum_{k=1}^{p} \sum_{n=1}^{\infty}\left\{\left[\frac{2(k-1)+n-\alpha}{1-\alpha}\right]\left|a_{n, p-k+1}\right|+\left[\frac{2(k-1)+n+\alpha}{1-\alpha}\right]\left|b_{n, p-k+1}\right|\right\} \\
& =1+\sum_{k=1}^{p}\left\{\sum_{n=1}^{\infty}\left\{\left|x_{n, p-k+1}\right|+\left|y_{n, p-k+1}\right|\right\}\right\} \\
& =1-\frac{1+\alpha}{1-\alpha}\left|b_{1,1}\right|-\sum_{k=2}^{p}\left\{\frac{2 k-1-\alpha}{1-\alpha}\left|a_{1, p-k+1}\right|+\frac{2 k-1+\alpha}{1-\alpha}\left|b_{1, p-k+1}\right|\right\} .
\end{aligned}
$$

The restriction placed in Theorem 3.1 on the moduli of the coefficients of $F=H+\bar{G}$ enables us to conclude for arbitrary rotation of the coefficients of $F$ that the resulting functions would still be harmonic univalent and $\mathrm{F} \in \mathcal{H} \mathcal{S}_{\mathrm{p}}(\alpha)$

Next, we discuss the geometric properties of mappings belonging to $\mathcal{H} \delta_{p}(\alpha)$.

Theorem 3.2. Each mapping in $\mathcal{H} \mathcal{S}_{p}(\alpha)$ maps $\mathbb{U}$ onto a starlike domain with respect to the origin.

Proof. Let $r \in(0,1)$ be a fixed number and

$$
F_{r}(z)=z+\sum_{n=2}^{\infty}\left(\sum_{k=1}^{p} r^{2(k-1)} a_{n, p-k+1}\right) z^{n}+\sum_{n=2}^{\infty}\left(\sum_{k=1}^{p} r^{2(k-1)} \overline{b_{n, p-k+1}}\right) \overline{z^{n}} .
$$

Obviously, $F_{r}$ is a harmonic mapping. Since

$$
\begin{aligned}
F_{r}(z) & =\sum_{n=2}^{\infty} n\left|\sum_{k=1}^{p} r^{2(k-1)} a_{n, p-k+1}\right|+\sum_{n=2}^{\infty} n\left|\sum_{k=1}^{p} r^{2(k-1)} b_{n, p-k+1}\right| \\
& \leqslant \sum_{n=2}^{\infty} \sum_{k=1}^{p}(2(k-1)+n)\left(\left|a_{n, p-k+1}\right|+\left|b_{n, p-k+1}\right|\right) \\
& \leqslant \sum_{k=1}^{p} \sum_{n=2}^{\infty}\left(\frac{2(k-1)+n-\alpha}{1-\alpha}\left|a_{n, p-k+1}\right|+\frac{2(k-1)+n-\alpha}{1-\alpha}\left|b_{n, p-k+1}\right|\right) \leqslant 1,
\end{aligned}
$$

it follows that $F_{r} \in \mathcal{H} \mathcal{S}_{p}(0)$. By (2.5), we know that $F_{r}$ maps $\mathbb{U}$ onto a starlike domain with respect to the origin for each $r \in(0,1)$, we show that $F$ is starlike with respect to the origin.

Example 3.3. Let $F_{1}(z)=z+\frac{1}{4} z^{2}+\frac{1}{8} \overline{z^{2}}$. Then $F_{1} \in \mathcal{H} \mathcal{S}_{1}\left(\frac{2}{7}\right)$ is a univalent, sense preserving polyharmonic mapping. In particular, $F_{1}$ maps $\mathbb{U}$ onto a starlike domain with respect to the origin (see Figure 1).

Example 3.4. Let $F_{2}(z)=z+\frac{1}{103} z^{2}+\frac{50}{103} \overline{z^{2}}$. Then $F_{2} \in \mathcal{H} \mathcal{S}_{1}\left(\frac{1}{52}\right)$ is a univalent, sense preserving polyharmonic mapping. In particular, $F_{1}$ maps $\mathbb{U}$ onto a starlike domain with respect to the origin (see Figure $1)$. 

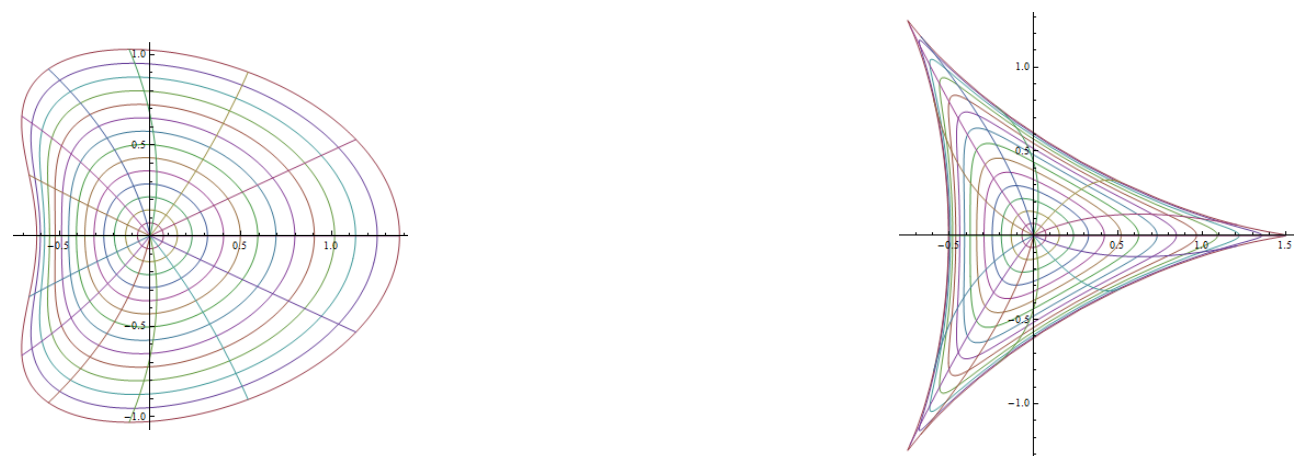

Figure 1: The images of $\mathbb{U}$ under the mappings $\mathrm{F}_{1}(z)=z+\frac{1}{4} z^{2}+\frac{1}{8} \overline{z^{2}}$ (left) and $\mathrm{F}_{2}(z)=z+\frac{1}{103} z^{2}+\frac{50}{103} \overline{z^{2}}$ (right).

We next show that the condition (3.1) is also necessary for functions in $\mathcal{H T}_{p}(\alpha)$.

Theorem 3.5. Let $\mathrm{F}=\mathrm{H}+\overline{\mathrm{G}}$ with $\mathrm{H}$ and $\mathrm{G}$ are given by (2.6). Then $\mathrm{F} \in \mathcal{H} \mathcal{T}_{\mathrm{p}}(\alpha)$ if and only if

$$
\begin{aligned}
& \sum_{k=1}^{p} \sum_{n=2}^{\infty}\left\{\left[\frac{2(k-1)+n-\alpha}{1-\alpha}\right]\left|a_{n, p-k+1}\right|+\left[\frac{2(k-1)+n+\alpha}{1-\alpha}\right]\left|b_{n, p-k+1}\right|\right\} \\
& \quad \leqslant 1-\frac{1+\alpha}{1-\alpha}\left|b_{1,1}\right|-\sum_{k=2}^{p}\left\{\frac{2 k-1-\alpha}{1-\alpha}\left|a_{n, p-k+1}\right|+\frac{2 k-1+\alpha}{1-\alpha}\left|b_{n, p-k+1}\right|\right\},
\end{aligned}
$$

where $0 \leqslant \frac{1+\alpha}{1-\alpha}\left|b_{1,1}\right|+\sum_{k=2}^{p}\left\{\frac{2 k-1-\alpha}{1-\alpha}\left|a_{n, p-k+1}\right|+\frac{2 k-1+\alpha}{1-\alpha}\left|b_{n, p-k+1}\right|\right\}<1$.

Proof. We first suppose that $F \in \mathcal{H} \mathcal{T}_{p}(\alpha)$, then by (2.5) we have

$$
\mathfrak{R}\left\{\frac{z \mathrm{H}^{\prime}(z)-\overline{\mathrm{G}^{\prime}(z)}}{\mathrm{H}(z)+\mathrm{G}(z)}\right\}-\alpha \geqslant 0 .
$$

The above condition must hold for all values of $z,|z|=r<1$. Upon choosing the values of $z$ on the positive real axis where $0 \leqslant z=r<1$ we must have

$$
\begin{aligned}
& \left(\left[(1-\alpha)-\left((1+\alpha)\left|b_{1, p}\right|\right)\right]-\sum_{k=2}^{p}\left\{(2 k-1-\alpha)\left|a_{n, p-k+1}\right|+(2 k-1+\alpha)\left|b_{n, p-k+1}\right| r^{2(k-1)}\right\}\right. \\
& \left.\quad-\sum_{k=1}^{p} \sum_{n=2}^{\infty}\left\{[2(k-1)+n-\alpha]\left|a_{n, p-k+1}\right|+[2(k-1)+n+\alpha]\left|b_{n, p-k+1}\right| r^{2 k+n-3}\right\} r^{2 k+n-3}\right) \\
& /\left(1-\sum_{k=2}^{p}\left\{\left|a_{1, p-k+1}\right|+\left|b_{1, p-k+1}\right| r^{2(k-1)}\right\}-\sum_{k=1}^{p} \sum_{n=2}^{\infty}\left\{\left|a_{n, p-k+1}\right|+\left|b_{n, p-k+1}\right|\right\} r^{2 k+n-3}\right) \geqslant 0 .
\end{aligned}
$$

If the condition (3.4) does not hold then the numerator in (3.5) is negative for $r$ sufficiently close to 1 . Thus there exits a $z_{0}=r_{0}$ in $(0,1)$ for which the quotient in (3.5) is negative. This contradicts the required condition for $F \in \mathcal{H T}_{p}(\alpha)$ and so the proof is complete.

Example 3.6. Let $\mathrm{F}_{1}(z)=z-\frac{1}{4} z^{2}+\frac{1}{8} \overline{z^{2}}$. Then $\mathrm{F}_{1} \in \mathcal{H} \mathcal{T}_{1}\left(\frac{10}{11}\right)$ is a univalent, sense preserving polyharmonic mapping. In particular, $F_{1}$ maps $\mathbb{U}$ onto a starlike domain with respect to the origin (see Figure 2).

Example 3.7. Let $F_{2}(z)=z-\frac{1}{103} z^{2}+\frac{50}{103} \overline{z^{2}}$. Then $F_{2} \in \mathcal{H} \mathcal{T}_{1}\left(\frac{5}{151}\right)$ is a univalent, sense preserving polyharmonic mapping. In particular, $F_{1}$ maps $\mathbb{U}$ onto a starlike domain with respect to the origin (see Figure 2). 

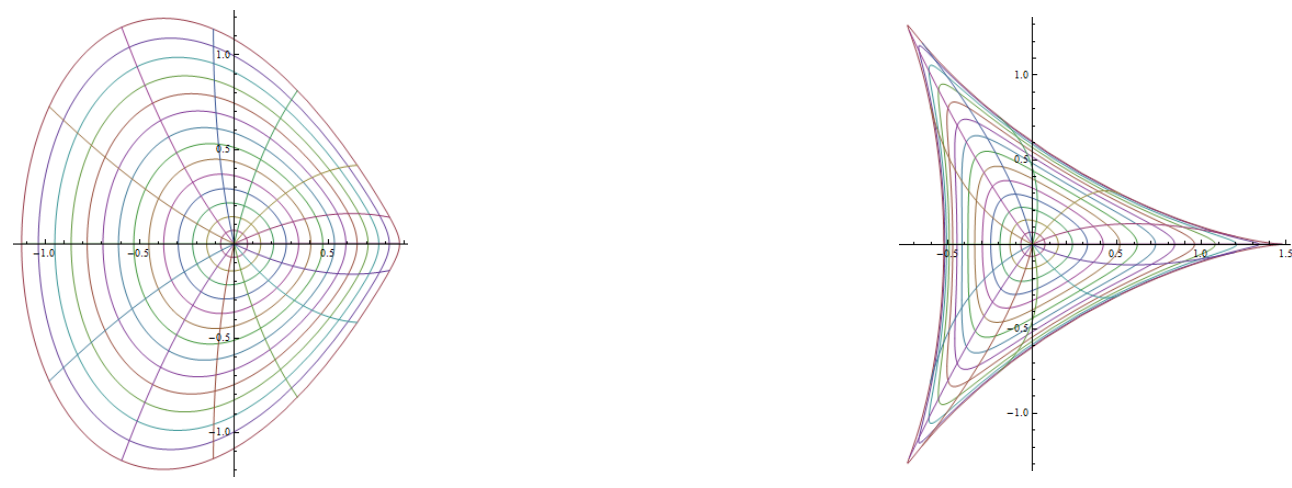

Figure 2: The images of $\mathbb{U}$ under the mappings $\mathrm{F}_{1}(z)=z-\frac{1}{4} z^{2}+\frac{1}{8} \overline{z^{2}}$ (left) and $\mathrm{F}_{2}(z)=z-\frac{1}{103} z^{2}+\frac{50}{103} \overline{z^{2}}$ (right).

Next, we shall obtain distortion bounds for functions in $\mathcal{H T}_{p}(\alpha)$ and also provide extreme points for the class $\mathcal{H T}_{\mathrm{p}}(\alpha)$.

Theorem 3.8. $\mathrm{F} \in \mathcal{H} \mathcal{T}_{\mathrm{p}}(\alpha)$ if and only if $\mathrm{F}$ can be expressed as

$$
\mathrm{F}(z)=\sum_{\mathrm{k}=1}^{\mathrm{p}} \sum_{n=1}^{\infty}\left(X_{\mathrm{n}, \mathrm{p}-\mathrm{k}+1} \mathrm{H}_{\mathrm{n}, \mathrm{p}-\mathrm{k}+1}(z)+\mathrm{Y}_{\mathrm{n}, \mathrm{p}-\mathrm{k}+1} \mathrm{G}_{\mathrm{n}, \mathrm{p}-\mathrm{k}+1}(z)\right),
$$

where

$$
\begin{aligned}
\mathrm{H}_{1,1}(z) & =z, \mathrm{H}_{\mathrm{n}, 1}(z)=z-\frac{1-\alpha}{n-\alpha} z^{\mathrm{n}} \quad(\mathrm{n}=2,3, \ldots), \\
\mathrm{H}_{\mathrm{n}, \mathrm{p}-\mathrm{k}+1}(z) & =z-|z|^{2(k-1)} \frac{1-\alpha}{2(k-2)+n-\alpha} z^{\mathrm{n}} \quad(\mathrm{n}=1,2, \ldots, 2 \leqslant k \leqslant p), \\
\mathrm{G}_{n, 1}(z) & =z+\frac{1-\alpha}{\mathrm{n}+\alpha} \overline{z^{n}}(\mathrm{n}=1,2, \ldots), \\
\mathrm{G}_{\mathrm{n}, \mathrm{p}-\mathrm{k}+1}(z) & =z+|z|^{2(k-1)} \frac{1-\alpha}{2(k-2)+n+\alpha} \overline{z^{n}} \quad(\mathrm{n}=1,2, \ldots, 2 \leqslant k \leqslant p),
\end{aligned}
$$

and

$$
\sum_{k=1}^{p} \sum_{n=1}^{\infty}\left(X_{n, p-k+1}+Y_{n, p-k+1}\right)=1,\left(X_{n, p-k+1} \geqslant 0, Y_{n, p-k+1} \geqslant 0\right) .
$$

In particular, the extreme points of $\mathcal{H} \mathcal{T}_{p}(\alpha)$ are $\left\{H_{n, p-k+1}\right\}$ and $\left\{G_{n, p-k+1}\right\}$.

Proof. Note that for F we may write

$$
\begin{aligned}
F(z)= & \sum_{k=1}^{p} \sum_{n=1}^{\infty}\left(X_{n, p-k+1} H_{n, p-k+1}(z)+Y_{n, p-k+1} G_{n, p-k+1}(z)\right) \\
= & \sum_{n=1}^{\infty} X_{n, 1} H_{n, 1}(z)+Y_{n, 1} G_{n, 1}(z)+\sum_{k=2}^{p} \sum_{n=1}^{\infty} X_{n, p-k+1} H_{n, p-k+1}(z)+Y_{n, p-k+1} \\
= & z-\sum_{k=2}^{p} \sum_{n=2}^{\infty}|z|^{2(k-1)} \frac{1-\alpha}{2(k-1)+n-\alpha} X_{n, p-k+1} z^{n} \\
& +\sum_{k=2}^{p} \sum_{n=1}^{\infty}|z|^{2(k-1)} \frac{1-\alpha}{2(k-1)+n+\alpha} Y_{n, p-k+1} \frac{z^{n}}{-} \sum_{n=2}^{\infty} \frac{1-\alpha}{n-\alpha} z^{n}+\sum_{n=1}^{\infty} \frac{1-\alpha}{n+\alpha} \frac{z^{n}}{}
\end{aligned}
$$


Then, by Theorem 3.5 we have

$$
\begin{aligned}
& \sum_{k=1}^{p} \sum_{n=2}^{\infty}\left\{\frac{1-\alpha}{2(k-1)+n-\alpha}\left(\frac{2(k-1)+n-\alpha}{1-\alpha} X_{n, p-k+1}\right)+\frac{1-\alpha}{2(k-1)+n+\alpha}\left(\frac{2(k-1)+n+\alpha}{1-\alpha} Y_{n, p-k+1}\right)\right\} \\
& \quad+Y_{1,1}+\sum_{k=2}^{p}\left(\frac{2 k-1-\alpha}{1-\alpha} \frac{1-\alpha}{2 k-1-\alpha} X_{1, p-k+1}+\frac{2 k-1+\alpha}{1-\alpha} \frac{1-\alpha}{2 k-1+\alpha} Y_{1, p-k+1}\right) \\
& \leqslant \sum_{k=1}^{p} \sum_{n=2}^{\infty}\left(X_{n, p-k+1}+Y_{n, p-k+1}\right)+\sum_{k=1}^{p}\left(X_{1, p-k+1}+Y_{1, p-k+1}\right)+Y_{1,1} \leqslant 1-Y_{1,1} \leqslant 1
\end{aligned}
$$

so $F \in \mathcal{H T}_{p}(\alpha)$. Conversely, suppose that $F \in \mathcal{H} \mathcal{T}_{p}(\alpha)$. Then

$$
\begin{aligned}
& \sum_{k=1}^{p} \sum_{n=2}^{\infty}\left\{\left[\frac{2(k-1)+n-\alpha}{1-\alpha}\right]\left|a_{n, p-k+1}\right|+\left[\frac{2(k-1)+n+\alpha}{1-\alpha}\right]\left|b_{n, p-k+1}\right|\right\} \\
& \leqslant 1-\frac{1+\alpha}{1-\alpha}\left|b_{1,1}\right|-\sum_{k=2}^{p}\left\{\frac{2 k-1-\alpha}{1-\alpha}\left|a_{n, p-k+1}\right|+\frac{2 k-1+\alpha}{1-\alpha}\left|b_{n, p-k+1}\right|\right\} .
\end{aligned}
$$

Setting

$$
\begin{aligned}
X_{n, p-k+1} & =\left(\frac{2(k-1)+n-\alpha}{1-\alpha}\right)\left|a_{n, p-k+1}\right|(2 \leqslant k \leqslant p, n=1,2, \ldots), \\
X_{n, 1} & =\left(\frac{n-\alpha}{1-\alpha}\right)\left|a_{n, 1}\right|(n=2,3, \ldots), \\
Y_{n, p-k+1} & =\left(\frac{2(k-1)+n+\alpha}{1-\alpha}\right)\left|b_{n, p-k+1}\right|(1 \leqslant k \leqslant p, n=1,2, \ldots),
\end{aligned}
$$

and

$$
X_{1,1}=1-\sum_{k=1}^{p} \sum_{n=2}^{\infty}\left(X_{n, p-k+1}+Y_{n, p-k+1}\right)-\sum_{k=2}^{p}\left(X_{1, p-k+1}+Y_{1, p-k+1}\right)-Y_{1,1}
$$

we obtain

$$
F(z)=\sum_{k=1}^{p} \sum_{n=1}^{\infty}\left(X_{n, p-k+1} H_{n, p-k+1}(z)+Y_{n, p-k+1} G_{n, p-k+1}(z)\right)
$$

as required.

Finally, we give the distortion bounds for functions in $\mathcal{H T}_{p}(\alpha)$, which yields a covering result for $\mathcal{H} \mathcal{T}_{p}(\alpha)$.

Theorem 3.9. If $\mathrm{F} \in \mathcal{H T}_{\mathrm{p}}(\alpha)$, then

$$
|f(z)| \leqslant\left(1+\left|b_{1,1}\right|\right) r+\left(\frac{1-\alpha}{2-\alpha}-\frac{1+\alpha}{2-\alpha}\left|b_{1,1}\right|\right) r^{2}, \quad|z|=r<1,
$$

and

$$
|f(z)| \geqslant\left(1-\left|b_{1,1}\right|\right) r-\left(\frac{1-\alpha}{2-\alpha}-\frac{1+\alpha}{2-\alpha}\left|b_{1,1}\right|\right) r^{2}, \quad|z|=r<1 .
$$


Proof. We only prove the first inequality. The argument for second inequality is similar and will be omitted. Let $F \in \mathcal{H T}_{p}(\alpha)$. Taking the absolute value of $F$, we obtain

$$
\begin{aligned}
|F(z)| \leqslant & \left(1+\left|b_{1,1}\right|\right)|z|+\left(\sum_{k=1}^{p} \sum_{n=2}^{\infty}\left(\left|a_{n, p-k+1}\right|+\left|b_{n, p-k+1}\right|\right)+\sum_{k=1}^{p}\left(\left|a_{1, p-k+1}\right|+\left|b_{1, p-k+1}\right|\right)\right)|z|^{n} \\
\leqslant & \left(1+\left|b_{1,1}\right|\right) r+\left(\sum_{k=1}^{p} \sum_{n=2}^{\infty}\left(\left|a_{n, p-k+1}\right|+\left|b_{n, p-k+1}\right|\right)+\sum_{k=1}^{p}\left(\left|a_{1, p-k+1}\right|+\left|b_{1, p-k+1}\right|\right)\right) r^{2} \\
= & \left(1+\left|b_{1,1}\right|\right) r+\frac{1-\alpha}{2-\alpha}\left(\sum_{k=1}^{p} \sum_{n=2}^{\infty}\left(\frac{1-\alpha}{2-\alpha}\left|a_{n, p-k+1}\right|+\frac{1-\alpha}{2-\alpha}\left|b_{n, p-k+1}\right|\right)\right. \\
& \left.+\sum_{k=1}^{p}\left(\frac{1-\alpha}{2-\alpha}\left|a_{1, p-k+1}\right|+\frac{1-\alpha}{2-\alpha}\left|b_{1, p-k+1}\right|\right)\right) r^{2} \\
\leqslant & \left(1+\left|b_{1,1}\right|\right) r+\frac{1-\alpha}{2-\alpha}\left(\sum_{k=1}^{p} \sum_{n=2}^{\infty}\left(\frac{2(k-1)+n-\alpha}{1-\alpha}\left|a_{n, p-k+1}\right|+\frac{2(k-1)+n+\alpha}{1-\alpha}\left|b_{n, p-k+1}\right|\right)\right. \\
& \left.+\sum_{k=1}^{p}\left(\frac{2 k-1-\alpha}{1-\alpha}\left|a_{1, p-k+1}\right|+\frac{2 k-1+\alpha}{1-\alpha}\left|b_{1, p-k+1}\right|\right)\right) r^{2} \\
\leqslant & \left(1+\left|b_{1,1}\right|\right) r+\frac{1-\alpha}{2-\alpha}\left(1-\frac{1+\alpha}{1-\alpha}\left|b_{1,1}\right|\right) r^{2} \quad(b y(3.4)) \\
= & \left(1+\left|b_{1,1}\right|\right) r+\left(\frac{1-\alpha}{2-\alpha}-\frac{1+\alpha}{2-\alpha}\left|b_{1,1}\right|\right) r^{2} .
\end{aligned}
$$

The bounds given in Theorem 3.5 for the functions $F=H+\bar{G}$ of the form (2.6) also hold for functions of the form (2.2) if the coefficient condition (3.1) is satisfied. The functions $F$ given by

$$
\mathrm{F}(z)=z+\left|\mathrm{b}_{1,1}\right| \bar{z}+\left(\frac{1-\alpha}{2-\alpha}-\frac{1+\alpha}{2-\alpha}\left|\mathrm{b}_{1,1}\right|\right) \overline{z^{2}} \text { and } \mathrm{F}(z)=z-\left|\mathrm{b}_{1,1}\right| z-\left(\frac{1-\alpha}{2-\alpha}-\frac{1+\alpha}{2-\alpha}\left|\mathrm{b}_{1,1}\right|\right) z^{2}
$$

for $\left|b_{1,1}\right| \leqslant(1-\alpha) /(1+\alpha)$ show that the bounds given in Theorem 3.5 are sharp.

The following covering result follows from the second inequality in Theorem 3.5.

Corollary 3.10. If $\mathrm{F} \in \mathcal{H T}_{\mathrm{p}}(\alpha)$, then

$$
\left\{w:|w|<\frac{1}{2-\alpha}\left(1-\left|\mathrm{b}_{1}\right|\right)\left[1+(2 \alpha-1)\left|\mathrm{b}_{1,1}\right|\right]\right\} \subset \mathrm{F}(\mathbb{U}) .
$$

The corresponding definition for polyharmonic convex function of order $\alpha$ leads to the following corollary.

Corollary 3.11. Let $\mathrm{F}$ be given by (2.1) and

$$
\begin{gathered}
\sum_{k=1}^{p} \sum_{n=2}^{\infty}\left\{\left[\frac{2(k-1)+n(n-\alpha)}{1-\alpha}\right]\left|a_{n, p-k+1}\right|+\left[\frac{2(k-1)+n(n+\alpha)}{1-\alpha}\right]\left|b_{n, p-k+1}\right|\right\} \\
\leqslant 1-\frac{1+\alpha}{1-\alpha}\left|b_{1,1}\right|-\sum_{k=2}^{p}\left\{\frac{2 k-1-\alpha}{1-\alpha}\left|a_{1, p-k+1}\right|+\frac{2 k-1+\alpha}{1-\alpha}\left|b_{1, p-k+1}\right|\right\},
\end{gathered}
$$

where $0 \leqslant \frac{1+\alpha}{1-\alpha}\left|\mathrm{b}_{1,1}\right|+\sum_{\mathrm{k}=2}^{\mathrm{p}}\left\{\frac{2 \mathrm{k}-1-\alpha}{1-\alpha}\left|\mathrm{a}_{1, \mathrm{p}-\mathrm{k}+1}\right|+\frac{2 \mathrm{k}-1+\alpha}{1-\alpha}\left|\mathrm{b}_{1, \mathrm{p}-\mathrm{k}+1}\right|\right\}<1$. Then $\mathrm{F}$ is univalent and sense preserving in $\mathbb{U}$ and $\mathrm{F} \in \mathcal{H} \mathcal{C}_{\mathrm{p}}(\alpha)$. 


\section{Acknowledgment}

The authors would like to thank University of Dammam and Deanship of Scientific Research at the University for there support to the present research.

\section{References}

[1] Y. Avci, E. Zlotkiewicz, On harmonic univalent mappings, Ann. Univ. Mariae Curie-Skodowska Sect. A, 44 (1990), 1-7.iii

[2] J. Clunie, T. Shell-Small, Harmonic univalent functions, Ann. Acad. Aci. Fenn. Ser. A I Math., 9 (1984), 3-25.1

[3] J. M. Jahangiri, Coefficient bounds and univalence criteria for harmonic functions with negative coefficients, Ann. Univ. Mariae Curie Sklodowska Sect A., 52 (1998), 57-66. 1

[4] J. M. Jahangiri, Harmonic functions starlike in the unit disk, J. Math. Anal. Appl., 235 (1999), 470-477.ii

[5] J. Qiao, X. Wang, On p-harmonic univalent mapping, Acta Math. Sci. A, 32 (2012), 588-600.2, i

[6] H. Silverman, Harmonic univalent functions with negative coefficients, Proc. Amer. Math. Soc., 51 (1998), 283-289.1

[7] H. Silverman, E. M. Silvia, Subclasses of harmonic univalent functions, New Zealand J. Math., 28 (1999), 275-284.1 